\title{
Selected electroweak results using $\tau$ leptons at BABAR
}

\author{
Elisa GUIDO** \\ INFN \& University of Genova, Via Dodecaneso 33, I-16146 Genova, Italy \\ E-mail: elisa.guido@ge.infn.it
}

A selection of electroweak measurements performed by the BABAR experiment operating at the SLAC National Accelerator Laboratory is presented. Using a sample of 122 million $\Upsilon(3 S)$ decays, we measure the ratio $R_{\tau \mu}=\operatorname{BR}\left(\Upsilon(1 S) \rightarrow \tau^{+} \tau^{-}\right) / \operatorname{BR}\left(\Upsilon(1 S) \rightarrow \mu^{+} \mu^{-}\right)$; the measurement is intended as a test of lepton universality and as a search for a light pseudoscalar Higgs boson in the Next to Minimal Supersymmetric Standard Model (NMSSM) scenario. Such a boson could appear in a deviation of the ratio $R_{\tau \mu}$ from the Standard Model expectation $\left(R_{\tau \mu} \sim 1\right)$. The analysis exploits the decays $\Upsilon(3 S) \rightarrow \Upsilon(1 S) \pi^{+} \pi^{-}, \Upsilon(1 S) \rightarrow l^{+} l^{-}$, where $l=\mu, \tau$. Two additional results are based instead on data recorded by $B A B A R$ at the $\Upsilon(4 S)$ resonance. The first one is a precision measurement of the mass of the tau lepton using $423 \mathrm{fb}^{-1}$ of data: using a pseudomass endpoint method, we determine the $\tau$ mass and we also measure the mass difference between the $\tau^{+}$and $\tau^{-}$. Finally, a precise measurement of the $\tau^{-} \rightarrow \bar{K}^{0} \pi^{-} v_{\tau}$ branching fraction is made using $\sim 384$ $\mathrm{fb}^{-1}$ of data.

European Physical Society Europhysics Conference on High Energy Physics, EPS-HEP 2009, July 16 - 222009

Krakow, Poland

* Speaker.

On behalf of the $B_{A} B_{A R}$ Collaboration 


\section{Introduction}

Despite it had been originally devoted to test CP-violation, the BABAR experiment (described elsewhere [1]) has obtained important results also in electroweak physics. Most of BABAR data has been collected at the $\Upsilon(4 S)$; at this energy the value of the cross section for the production of $\tau^{+} \tau^{-}$pairs $\left(\sigma\left(e^{+} e^{-} \rightarrow \tau^{+} \tau^{-}\right) \sim 0.92 \mathrm{nb}\right)$ is pretty near to the value for the production of $b \bar{b}$ pairs $\left(\sigma\left(e^{+} e^{-} \rightarrow b \bar{b}\right) \sim 1.05 \mathrm{nb}\right)$. Therefore, it is clear that BABAR can be a very useful laboratory where to investigate $\tau$ physics as well. Here I will show two results: a precision measurement of the mass of the $\tau$ lepton and the measurement of the $\tau^{-} \rightarrow \bar{K}^{0} \pi^{-} v_{\tau}$ branching fraction [2]. Moreover, important electroweak tests can be performed on the large datasets collected by BABAR at lower energies in the center of mass; in particular the result shown here is obtained using the $\sim 28 \mathrm{fb}^{-1}$ of data collected at the $\Upsilon(3 S)$ energy and is intended as a test of lepton universality in $\Upsilon(1 S)$ decays, by measuring the ratio $R_{\tau \mu}=\operatorname{BR}\left(\Upsilon(1 S) \rightarrow \tau^{+} \tau^{-}\right) / \operatorname{BR}\left(\Upsilon(1 S) \rightarrow \mu^{+} \mu^{-}\right)$.

\section{Test of lepton universality using $\Upsilon(1 S)$ decays}

In the Standard Model (SM) the couplings between gauge bosons and leptons are independent of lepton flavor; therefore the quantity $R_{l l^{\prime}}=\frac{B R\left(\Upsilon(1 S) \rightarrow l^{+} l^{-}\right)}{B R\left(\Upsilon(1 S) \rightarrow l^{\prime} l^{\prime-}\right)}$, where $l, l^{\prime}=e, \mu, \tau$, is expected to be very near to one, the discrepancy from unity being due to small lepton-mass effects (for instance, the greatest correction should turn out in $R_{\tau \mu} \sim 0.992$ ).

In the NMSSM [3] deviations of $R_{l l^{\prime}}$ from the SM expectation are possible, in the hypothesis of existence of a light CP-odd Higgs boson, namely $A^{0}$. If its mass was just beneath the $\Upsilon(1 S)$ mass, it could enter the decay chain of the resonance $[4]^{1}$ :

$$
\Upsilon(1 S) \rightarrow A^{0} \gamma, A^{0} \rightarrow l^{+} l^{-} \quad \text { or } \quad \Upsilon(1 S) \rightarrow \eta_{b} \gamma, \eta_{b} \rightarrow A^{0} \rightarrow l^{+} l^{-} .
$$

If the photon remained undetected, the lepton pair would be ascribed to the $\Upsilon(1 S)$, resulting in a lepton universality violation. The coupling between $A^{0}$ and the lepton being proportional to the lepton mass, this effect should be more evident when one of the leptons is a $\tau$. The search made by BABAR considers $R_{\tau \mu}$, exploiting the $\sim 28 \mathrm{fb}^{-1}$ statistics collected at the $\Upsilon(3 S)$ energy. The signal events $\left(\Upsilon(1 S) \rightarrow \mu^{+} \mu^{-}\right.$or $\left.\tau^{+} \tau^{-}\right)$are tagged through the transition $\Upsilon(3 S) \rightarrow \Upsilon(1 S) \pi^{+} \pi^{-}$, and considering all the decays of $\tau$ to one charged track plus undetected neutrals. This choice leads to a very clear environment for our study, since the final state contains four charged tracks, allowing for a better handling of backgrounds and a good trigger performance. The event selections are tuned using Monte Carlo simulated samples and considering separately $\Upsilon(1 S) \rightarrow \mu^{+} \mu^{-}$and $\Upsilon(1 S) \rightarrow$ $\tau^{+} \tau^{-}$events, because of different levels of backgrounds. The signal extraction is performed using an extended and unbinned maximum-likelihood fit; the likelihood for $\Upsilon(1 S) \rightarrow \mu^{+} \mu^{-}$events is based on the variables $\Delta M=M_{\Upsilon(3 S)}-M_{\Upsilon(1 S)}$ and $M_{\mu^{+} \mu^{-}}$, while for $\Upsilon(1 S) \rightarrow \tau^{+} \tau^{-}$events the recoil mass of the two leptons is used, $M_{\pi^{+} \pi^{-}}^{\text {reco }}=\sqrt{s+M_{\pi^{+} \pi^{-}}^{2}-2 \cdot \sqrt{s} \cdot \sqrt{M_{\pi^{+} \pi^{-}}^{2}+p_{\pi^{+} \pi^{-} C M}^{2}}}$, where $\sqrt{s}$ indicates the energy of data acquisition. The fit is performed simultaneously to the two kinds of events, with the results shown in Figure 1 . The value of $R_{\tau \mu}$ returned by the fit is then corrected for

\footnotetext{
${ }^{1}$ The latter decay implies a mixing between $\eta_{b}$, i.e. the ground state of the bottomonium family, recently discovered by BABAR [5], and which does not have the right quantum numbers to decay in a pair of leptons, and the light Higgs.
} 

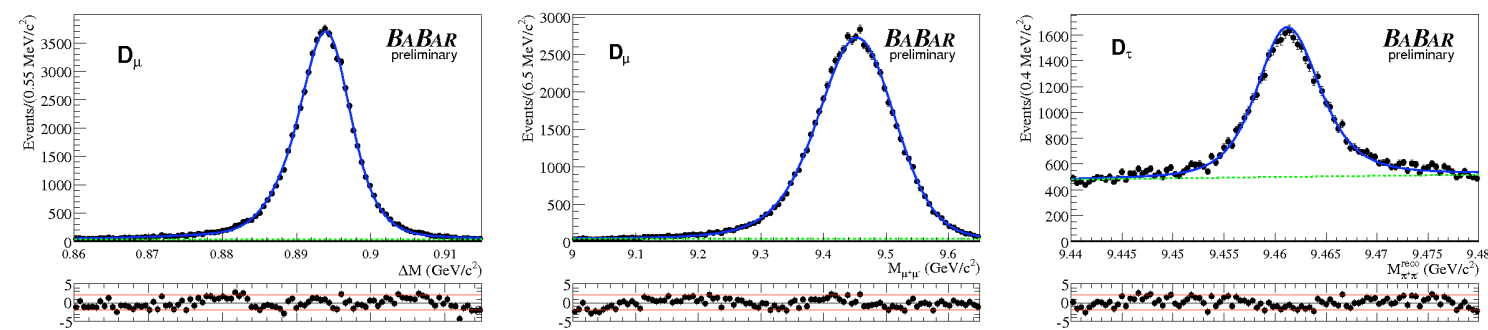

Figure 1: Results of the fit procedure for the $\Delta M$ distribution (left), the $M_{\mu^{+} \mu^{-}}$distribution (center) and for the $M_{\pi^{+}}^{\text {reco }} \pi^{-}$ distribution (right). In each plot, the dashed green line represents the background shape, while the solid blue line is the sum of signal and bakground contributions. The residuals of the fit are shown under each plot, with the solid red lines indicating the $2 \sigma$ level of agreement. $D_{\mu}$ and $D_{\tau}$ indicate the kind of events $\left(\Upsilon(1 S) \rightarrow \mu^{+} \mu^{-}\right.$or $\left.\tau^{+} \tau^{-}\right)$which each plot refers to.

known differences between data and simulation, and an estimate of the systematic uncertainty is made: the main contributions arise from the differences between data and simulation in the event selection, the particle identification and the trigger efficiency, and from the imperfect knowledge of signal and background shapes used in the fit. The preliminary result obtained by BABAR is:

$$
R_{\tau \mu}=1.009 \pm 0.010 \text { (stat.) } \pm 0.024 \text { (syst.). }
$$

This result improves upon the previous best result obtained by the CLEO Collaboration [6], both in statistical and in systematic precision. No significant deviation with respect to the SM expectation is observed.

\section{Precision measurement of $\tau$ mass and $\tau^{+}-\tau^{-}$mass difference}

The measurement of the difference in mass between a particle and its anti-particle is a common test of $C P T$ invariance for the SM. In BABAR this can be performed measuring the masses of the $\tau$ leptons. The analysis exploits the pseudomass endpoint method [7, 8]: considering the hadronic decays $\tau^{ \pm} \rightarrow h^{ \pm} v_{\tau}$, where $h$ indicates the total hadronic system of the final state, one can define the $\tau$ mass as $M_{\tau}=\sqrt{M_{h}^{2}+2\left(\sqrt{s} / 2-E_{h}^{*}\right)\left(E_{h}^{*}-p_{h}^{*} \cos \theta^{*}\right)}$, where $M_{h}, E_{h}^{*}$ and $p_{h}^{*}$ are the invariant mass, the energy calculated in the center of mass frame and the momentum in the same frame of the hadronic system, respectively. The neutrino, whose momentum direction forms an angle $\theta^{*}$ with the momentum of $h$ in the center of mass frame, escapes undetected, making impossible the reconstruction of $M_{\tau}$. The pseudomass $M_{p}$ can thus be defined as the lower limit of $M_{\tau}$ for $\theta^{*}=0$ : $M_{p}=\sqrt{M_{h}^{2}+2\left(\sqrt{s} / 2-E_{h}^{*}\right)\left(E_{h}^{*}-p_{h}^{*}\right)}$. The $M_{p}$ distribution has a sharp kinematic cutoff at $M_{p}=$ $M_{\tau}$, despite a smearing effect, due to initial and final state radiation, as well as to detector finite resolution. The measurement uses the $432 \mathrm{fb}^{-1}$ statistics collected by BABAR at the $\Upsilon(4 S)$ energy, correspondent to $\sim 4 \cdot 10^{8} \tau^{+} \tau^{-}$pairs. We consider as signal events the decays $\tau^{ \pm} \rightarrow \pi^{ \pm} \pi^{+} \pi^{-} v_{\tau}$. The presence of a lepton, coming from the decay of the other $\tau$, in the hemisphere opposite to the signal is also required. The relation between the $M_{p}$ endpoint and $M_{\tau}$ is needed to get $M_{\tau}$ from the endpoint fit to data, shown in Figure 3; it is determined using Monte Carlo simulated samples, generated with different values of $M_{\tau}$. This relation is found to be linear with a slope of unity, as expected, but with a non zero offset, due to imperfect detector resolution. The data are split in 


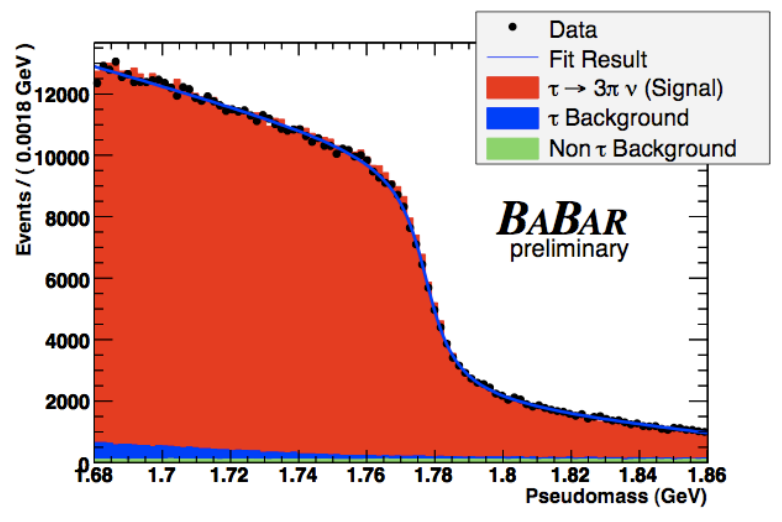

Figure 2: Average $M_{p}$ distribution. The histogram is the simulation, dots are data and the curve is the fit to the data.

two samples, according to the total charge of the $3 \pi$-system, in order to perform both an average and a separate measurement of $M_{\tau^{+}}$and $M_{\tau^{-}}$. Possible effects of bad reconstruction of tracks momenta, which is the greatest contribution to the systematic uncertainty, are carefully considered. The BABAR preliminary results, in good agreement with the world average [9], are:

$$
\begin{gathered}
M_{\tau}=(1776.68 \pm 0.12 \text { (stat.) } \pm 0.41 \text { (syst.) }) \mathrm{MeV} / \mathrm{c}^{2} \\
\left.\left.\frac{M_{\tau^{+}}-M_{\tau^{-}}}{M_{\text {average }}}=(-3.5 \pm 1.3 \text { (stat. }) \pm 0.3 \text { (syst. }\right)\right) \cdot 10^{-4} .
\end{gathered}
$$

\section{Measurement of $\tau^{-} \rightarrow K_{S}^{0} \pi^{-} v_{\tau}$ branching fraction}

The hadronic $\tau$ decays are useful for studying the weak currents, since they give access both to the vector and to the axial-vector spectral functions. Hadrons from $\tau$ decays are produced via $W$ emission; strangeness-changing $\tau$ decays are suppressed and resonant decay dominates these currents. BABAR measures the $B F\left(\tau^{-} \rightarrow \bar{K}^{0} \pi^{-} v_{\tau}\right)$ exploiting a $385 \mathrm{fb}^{-1}$ statistics collected at the $\Upsilon(4 S)$ energy, only looking for $\tau^{-} \rightarrow K_{S}^{0} \pi^{-} v_{\tau}$, with $K_{S}^{0} \rightarrow \pi^{+} \pi^{-}$final states. The events are well separated in two hemispheres: the signal side, where three pions are reconstructed, and the tag side, where the presence of one lepton from the $\tau$ decay is required. The main background contributions are non- $\tau$, Bhabha and $\tau^{-} \rightarrow \pi^{-} \pi^{+} \pi^{-} \nu_{\tau}$ events, powerfully rejected by the event selection, and irreducible $\tau^{-} \rightarrow K_{L}^{0} K_{S}^{0} \pi^{-} v_{\tau}$ and $\tau^{-} \rightarrow \pi^{0} K_{S}^{0} \pi^{-} \nu_{\tau}$ events, with the additional neutrals undetected. After the selection, the signal efficiency is $\sim 1.1 \%$. The main contributions to the systematic uncertainty arise from the particle identification and the tracking efficiency; the total uncertainty is of $2.72 \%$. The preliminary result obtained by $B A B A R$ for the branching fraction is [2]:

$$
B F\left(\tau^{-} \rightarrow \bar{K}^{0} \pi^{-} v_{\tau}\right)=0.840 \pm 0.004 \text { (stat.) } \pm 0.023 \text { (syst.). }
$$

This result is compatible with the previous one obtained by the Belle Collaboration [10] with a comparable statistics; the systematic precision of the two analyses is also comparable. 


\section{Conclusions}

The results shown here are a clear evidence that $B A B A R$ data are a rich harvest not only for b-physics but for electroweak physics as well, allowing for several important, and different, tests of the Standard Model. The finalization of these analyses, expected for soon, promises a further improvement in the precision.

\section{References}

[1] B. Aubert et al. [The BABAR Collaboration], The BABAR detector, Nucl. Instrum. Meth. A 479 (1) $2002[$ hep-ex/ 0105044$]$.

[2] B. Aubert et al. [The BABAR Collaboration], Measurement of the branching fraction $B\left(\tau \rightarrow K^{0} \pi^{-} v\right)$ using the BaBar detector, hep-ex/0808.1121.

[3] F. Gunion et al., The Higgs Hunter's Guide, Addison-Wesley Publishing Company, 1990.

[4] M. A. Sanchiz-Lozano, Leptonic universality breaking in Upsilon decays as a probe of new physics, Int. J. Mod. Phys. A 19 (2183) 2004 [hep-ph / 0307313$]$;

E. Fullana and M. A. Sanchiz-Lozano, Hunting a light CP-odd non-standard Higgs boson through its tauonic decay at a (Super) B factory, Phys. Lett. B 653 (67) 2007 [hep-ph / 0702190 ].

[5] B. Aubert et al. [The BABAR Collaboration], Observation of the bottomonium ground state in the decay $\Upsilon(3 S) \rightarrow \gamma \eta_{b}$, Phys. Rev. Lett.101 (071801) 2008 [hep-ex/0807.1086].

[6] D. Besson et al. [The CLEO Collaboration], First Observation of $\Upsilon(3 S) \rightarrow \tau \tau$ and Tests of Lepton Universality in Upsilon Decays, Phys. Rev. Lett. 98 (052002) 2007 [hep-ex/ 0607019 ].

[7] H. Albrecht et al. [The ARGUS Collaboration], A Measurement of the tau mass, Phys. Lett. B 292 (221) 1992.

[8] K. Abe et al. [The Belle Collaboration], Measurement of the mass of the $\tau$-lepton and an upper limit on the mass difference between $\tau^{+}$and $\tau^{-}$, Phys. Rev. Lett. 99 (011801) 2007 [hep-ex/ 0608046 ].

[9] C. Amsler et al. [Particle Data Group], Review of Particle Physics, Phys. Lett. B 667 (1) 2008.

[10] D. Epifanov et al. [The Belle Collaboration], Study of $\tau \rightarrow K_{S} \pi^{-} v_{\tau}$ decay at Belle, Phys. Lett. $B \mathbf{6 5 4}$ (65) 2007 [hep-ex/0706.2231]. 